Bundesgesundheitsbl 2018 · 61:1604

https://doi.org/10.1007/s00103-018-2829-5

(c) Springer-Verlag GmbH Deutschland, ein Teil von Springer Nature 2018

Bekanntmachung des Umweltbundesamtes

\title{
Neuberufung der Mitglieder der Trinkwasserkommission beim Umweltbundesamt
}

- Frau Dr. Ulrike Schuhmacher-Wolz, Forschungs- und Beratungsinstitut Gefahrstoffe GmbH, Freiburg,

- Frau Dr. Karlin Stark, Regierungspräsidium Stuttgart, Landesgesundheitsamt,

- Herrn Dr. Roland Suchenwirth, Niedersächsisches Landesgesundheitsamt, Hannover,

- Frau Dipl.-Ing. Kerstin Voigt, Amt für Gesundheit, Frankfurt am Main,

- Frau Dr. Gerlinde Wauer, Landesamt für Gesundheit und Soziales Mecklenburg-Vorpommern, Neustrelitz,

- Herrn Dipl.-Biol. Hartmut Willmitzer, Thüringer Fernwasserversorgung, Erfurt und

- Herrn Dr.-Ing. Burkhard Wricke, Technologiezentrum Wasser, Außenstelle Dresden.

Die Kommissionsmitglieder wählten Frau Prof. Dr. Christiane Höller zur Vorsitzenden und Frau Dipl.-Chem. Uta Rädel zur stellvertretenden Vorsitzenden der Trinkwasserkommission.

Die Trinkwasserkommission hat die Aufgabe, das Umweltbundesamt und das Bundesministerium für Gesundheit insbesondere bei der Entwicklung von Konzeptionen zur Vorbeugung, Erkennung und Verhinderung der Weiterverbreitung von durch Wasser übertragbaren Krankheiten $\mathrm{zu}$ beraten und zu unterstützen. Dazu erarbeitet sie wissenschaftlich begründete Stellungnahmen und Empfehlungen zum Schutz der menschlichen Gesundheit hinsichtlich der Anforderungen an die Qualität von Trinkwasser im Hinblick auf die mikrobiologische und chemische Beschaffenheit sowie der insoweit notwendigen Maßnahmen und berücksichtigt dabei Belange der praktischen Durchführung.

Der wissenschaftlichen Geschäftsstelle beim Umweltbundesamt ist die Organisation der Kommissionsarbeit übertragen worden.

Laut Geschäftsordnung können an den Sitzungen der Trinkwasserkommission (als nicht stimmberechtigt) Vertreterinnen und Vertreter des Bundesministeriums für Gesundheit, des Bundesministeriums für Umwelt, Naturschutz und nukleare Sicherheit, des Bundesministeriums für Verteidigung, des Umweltbundesamtes und der zuständigen Landesbehörden teilnehmen.

(Siehe auch unter http://www.umweltbundesamt.de/themen/wasser/trinkwasser/trinkwasserkommission) 